\title{
Erratum to: Imaging findings of anaplastic astrocytoma in a child with maple syrup urine disease: a case report
}

\author{
Jessie Aw-Zoretic ${ }^{1,4}$ - Nitin R. Wadhwani ${ }^{2,5}$ • Rishi R. Lulla ${ }^{3,6}$ • Maura E. Ryan ${ }^{1,4}$
}

Published online: 16 July 2015

(C) Springer-Verlag Berlin Heidelberg 2015

\section{Erratum to: Child'Nervous System}

DOI 10.1007/s00381-015-2756-9

The original publication of this article unfortunately captured one of the authors' name incorrectly.

Instead of Lulla R. Rishi it should have been Rishi R. Lulla.

Jessie Aw-Zoretic

jawdoc3@gmail.com

Rishi R. Lulla

rlulla@luriechildrens.org

Maura E. Ryan

mryan@luriechildrens.org

1 Department of Medical Imaging, Ann \& Robert H. Lurie Children's Hospital, Chicago, IL, USA

2 Department of Pathology, Ann \& Robert H. Lurie Children's Hospital, Chicago, IL, USA
3 Division of Hematology, Oncology, Neuro-Oncology and Stem Cell Transplant, Ann \& Robert H. Lurie Children's Hospital, Chicago, IL, USA

4 Department of Radiology, Northwestern University Feinberg School of Medicine, Chicago, IL, USA

5 Department of Pathology, Northwestern University Feinberg School of Medicine, Chicago, IL, USA

6 Department of Pediatrics, Northwestern University Feinberg School of Medicine, Chicago, IL, USA 